\title{
Metal-Semiconductor Transition on the Surface and in the Bulk of Europium Hydride Thin Film
}

\author{
M. Knor, R. Nowakowski* and R. Duś \\ Institute of Physical Chemistry, Polish Academy of Sciences, M. Kasprzaka 44/52, 01-224 Warszawa, Poland
}

(Received April 17, 2012; in final form August 30, 2012)

\begin{abstract}
Thin europium films $(20-50 \mathrm{~nm}$ thick $)$ on a glass substrate were transformed into $\mathrm{EuH}_{x}(0<x<2)$ by interaction with $\mathrm{H}_{2}$ introduced into the reactor in successive calibrated doses. By measuring the pressure, the hydrogen uptake $(\mathrm{H} / \mathrm{Eu})$ was determined at every step of the reaction. In situ monitoring of bulk properties (electrical resistance $R(\mathrm{H} / \mathrm{Eu})$, relative transparency to light $T(\mathrm{H} / \mathrm{Eu}) / T_{0}$ and $(\mathrm{H} / \mathrm{Eu})$ dependent light transparency spectrum) confirms metal-semiconductor transition at room temperature. Both the electrical resistance and optical transparency of the film strongly increase with hydrogen concentration as a consequence of the resulting increase of the content of semiconducting dihydride. Moreover, the course of work function changes $\Delta \Phi(\mathrm{H} / \mathrm{Eu})$ indicates inversion of the charge-transfer direction on the surface. The transition at room temperature from positively to negatively polarized hydrogen adsorbate was observed in situ during hydrogen uptake. As a result, the work function at equilibrium state varies with hydrogen content from +18 to $-18 \mathrm{mV}$ with respect to pure metal film, reflecting the change of "mirror potential" generated on the surface due to the accumulation of hydrogen adsorbates in the subsurface region.
\end{abstract}

PACS: 67.63.Gh, 73.61.-r, 78.66.-w, 73.20.-r

\section{Introduction}

$\mathrm{H}_{2}$ interaction with rare earth metals (RE) leads to the formation of hydrides when appropriate thermodynamic conditions are fulfilled. This reaction is accompanied by the transition of the light-reflecting original metal into a transparent semiconductor [1-3]. Studies of metal-semiconductor transition caused by RE interaction with hydrogen are very interesting from the point of view of basic research (correlated changes of electrical, optical, magnetic properties, hydrogen diffusion, isotopic effect) and also practical applications. These materials, often called switchable mirrors, offer potential possibilities for application in novel electro-optical devices like smart windows, optical shutters, displays, gas sensors.

The majority of investigations have been concerned with bulk properties [4-7]. On the other hand, surface phenomena associated with this transition are still poorly recognized. One of the important reasons for this situation is the high reactivity of $\mathrm{RE}$ metals which causes experimental difficulties with maintaining a clean surface and measuring its properties during interaction with hydrogen. Thus the majority of described investigations were performed ex situ and concerned bilayer systems in which RE film is protected against oxidation by a thin palladium overcoat. This solution has been applied in studies of bulk phenomena (electrical and optical properties) associated with hydride formation in thin films of several hexagonal metals, like Y [8], Gd [9], Tb [10], Ho [11]. We have recently performed in situ measurements of uncovered thin Gd [12] and Tb [13] films during interaction with hydrogen, extending investigations into

* corresponding author; e-mail: rnowakowski@ichf .edu.pl surface phenomena using the static capacitor method. Moreover, comparison of basic differences in thin films response on hydride formation between $\mathrm{Tb}$ and $\mathrm{Eu}$ has been undertaken [14].

In this article, we report more detailed studies on thin films of europium. For both basic and applied aspects it is of great interest to study hydrogen interaction with europium due to special electron configuration of this metal and its unique structural, optical and magnetic properties during hydrogenation. The previously investigated metals belong to the main group of hexagonal RE elements which form three $\mathrm{REH}_{x}$ phases at room temperature: solid solution of hydrogen in the host-metal lattice ( $\alpha$ phase), metallic fcc dihydrides ( $\beta$ phase) and hexagonal lattice semiconductor trihydrides ( $\gamma$ phase). Europium is characterized by very different properties as a pure metal (bcc crystal structure) and as europium hydride, as it forms solid solution of hydrogen ( $\alpha$ phase) and a characteristic for this metal semiconductor dihydride. The latter is crystallized into orthorhombic structure $\left(\mathrm{PbCl}_{2}\right.$-type, see $[15,16]$ for crystallographic data). It is worth to emphasize that other $\mathrm{EuH}_{x}$ phases have not been determined in hydrogen atmosphere even at higher temperatures. Recently, Matsuoka et al. [17] reported interesting results showing further $\mathrm{EuH}_{x}$ structural transitions associated with $\mathrm{Eu}^{2+}$ to $\mathrm{Eu}^{3+}$ valence changes, however there were induced by reaction with high-pressure hydrogen (7.2-9.7 GPa).

Optical properties of thin films of europium dihydride covered by protected palladium cap layer were investigated ex situ in the infrared and visible spectral ranges by Weber and Schoenes [18]. The optical band gap was determined by transmission measurements and discussed with respect to electronic structure of $\mathrm{EuH}_{2}$ (assigned to transition $4 f^{7}$ to $5 d$ ). 
The aim of this investigations is to find the correlation between elementary steps of surface processes and bulk property changes caused by hydride formation in thin europium film as a function hydrogen content $(0<$ $\mathrm{H} / \mathrm{Eu}<2)$. In situ measurements of work function changes $\Delta \Phi(\mathrm{H} / \mathrm{Eu})$ were chosen to illustrate the surface phenomena, while changes of the electrical resistance $R(\mathrm{H} / \mathrm{Eu})$, relative light transmittance $T(\mathrm{H} / \mathrm{Eu}) / T_{0}$ and the light absorption spectrum correspond to bulk properties.

\section{Experimental}

The experiments were performed using a UHV glass system capable of routinely reaching the basis static pressure of $1 \times 10^{-8} \mathrm{~Pa}$. It is worth remembering that in glass UHV systems evacuated by mercury pumps nitrogen is the main component of the residual gases. Thus the hydrogen partial pressure was of the order of $10^{-10} \mathrm{~Pa}$, enabling studies of europium interaction with hydrogen under pressure of $10^{-8} \mathrm{~Pa}$.

\subsection{Eu film preparation, electrical resistance and optical transmittance measurements}

Thin films were obtained by evaporation of strips of Eu foil (Alfa Aesar, Johnson Matthey GmbH, 99.9\%) from a tungsten heater, and their deposition on the wall of the reactor made of Pyrex glass, maintained at a constant temperature. The amount of $\mathrm{Eu}$ in the films was precisely determined by the spectrophotometric method using metal complexation with the dye molecule Arsenazo III (Sigma-Aldrich Co.). In this procedure the films after experiments were firstly dissolved in a $\mathrm{HCl}$ solution, then the dye molecules were added for complexation and the obtained solutions were analyzed spectrophotometrically. The spherical reactor was equipped with two electrodes made of platinum foil, semi-melted onto the glass of the cell and connected to the tungsten feedthroughs. This allowed electrical contact with the film during its deposition and interaction with hydrogen. The resistance of the Eu films ranged between $40-100 \Omega$ depending on the film thickness (50-20 nm, respectively). The electrical resistance and optical transparency changes of the film caused by the interaction with hydrogen were measured in situ during introduction of successive calibrated doses of spectroscopically pure hydrogen, additionally purified by diffusion through a palladium thimble, into the reactor separated from the pumps. The change of $\mathrm{H}_{2}$ pressure was monitored continuously by means of a Pirani gauge allowing to determine the amount of consumed hydrogen for each dose. Using this procedure the atomic ratio $\mathrm{H} / \mathrm{Eu}$ was calculated for each step of $\mathrm{EuH}_{x}$ formation. The resistance of the thin film was continuously measured within the interval $10^{-3}-10^{10} \Omega$. The changes in light absorbance spectrum were simultaneously monitored in situ within the wavelength $\lambda=200-1100 \mathrm{~nm}$ by the location of the glass reactor directly in the measurement path of the UV-1202 Shimadzu spectrophotometer. The integration of spectra allows to calculate the change of the film transmittance $T(\mathrm{H} / \mathrm{Eu})$ caused by hydride formation.

\subsection{Work function measurements}

Surface phenomena were studied by measurement of work function changes $\Delta \Phi$ using a home-made static capacitor circuit (sensitivity $1 \mathrm{mV}$, response time $1 \mathrm{~ms}$, and stability $1 \mathrm{mV} / \mathrm{h}$ ) [19-21]. The active electrode was formed by the deposition of analogous Eu film as used for the electrical and optical measurements. The reference electrode was obtained by melting a conducting coating of $\left(\mathrm{SnO}+\mathrm{Sb}_{2} \mathrm{O}_{5}\right)$ into glass. The courses of $\Delta \Phi(t)$ were continuously registered following each successive hydrogen dose introduction into the apparatus separated from the pumps. The work function isotherm $\Phi=f(\mathrm{H} / \mathrm{Eu})_{T}$ can then be constructed from $\Delta \Phi$ changes determined at equilibrium after introduction of each $\mathrm{H}_{2}$ dose.

\section{Results and discussion}

\subsection{Electrical resistance and optical transmittance changes}

Figure 1 presents photographs of the glass reactor with thin $\mathrm{Eu}$ film (50 nm thick) (a) before and (b) after $\mathrm{H}_{2}$ $(30 \mathrm{~Pa})$ introduction at room temperature. The transition of the light-reflecting pure Eu film into transparent orange $\mathrm{EuH}_{2}$ film is evident. Decreasing the temperature of the reactor with $\mathrm{EuH}_{2}$ film resulted in an increase of the film's resistance, confirming its semiconductor character. This metal-semiconductor transition is reversible. The evacuation of hydrogen over $\mathrm{EuH}_{2}$ film leads to a loss of the film's optical transparency and restores the positive temperature coefficient of electrical resistance typical for pure metal. We can therefore conclude that thin Eu film in UHV conditions shows switchable optical properties at $298 \mathrm{~K}$ during in situ interaction with an $\mathrm{H}_{2}$ atmosphere. The changes of optical and electrical properties of the $\mathrm{Eu}$ film agree with those reported before for $\beta-\gamma$ transition within $\mathrm{YH}_{x}$ [22], $\mathrm{TbH}_{x}$ [13], $\mathrm{GdH}_{x}(2<x<3)[12]$. This indicates that the metalsemiconductor transition caused by hydride formation is not a characteristic property only of the main group of $\mathrm{RE}$ hexagonal metals but is a common property of RE elements of different crystallographic structures.

Figure $1 c$ shows the set of optical absorbance spectra $A_{\mathrm{H} / \mathrm{Eu}}(\lambda)$ determined in situ for selected hydrogen contents in uncapped hydride films under equilibrium conditions reached after successive $\mathrm{H}_{2}$ dose introduction. Variation of the absorbance course, reflecting the change of the electronic structure of the hydrides with increasing hydrogen concentration, is clearly visible. The absorbance of pure Eu film (spectrum (0) from the top) is characterized by a minimum for wavelength around $390 \mathrm{~nm}$. This is the reason for the blue-metallic color of thin pure Eu film. At the same time, the maximal absorbance is noted in a low-energy region (near infrared). This part of the spectrum is most sensitive during hydride 


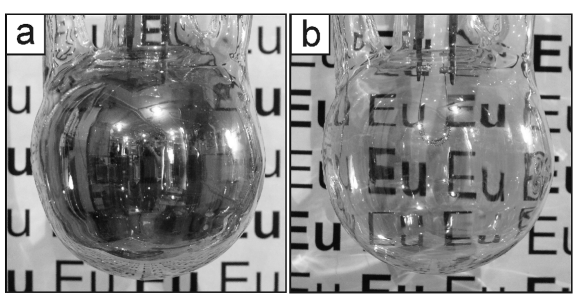

absorbance

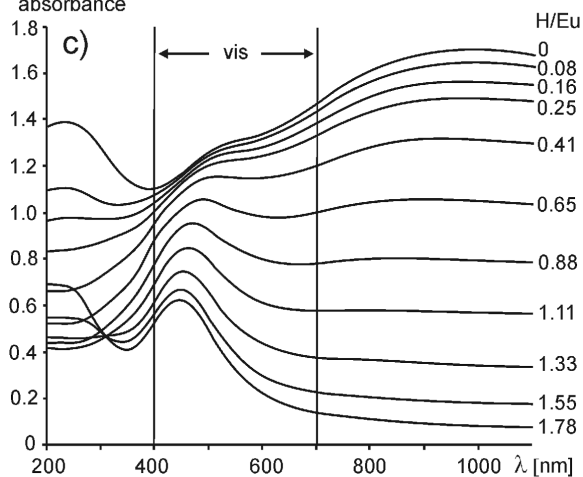

Fig. 1. Change of optical transparency of thin Eu film during $\mathrm{EuH}_{x}(0<x<2)$ formation: (a) a reactor with metallic, light-reflecting thin Eu film, (b) a reactor with transparent thin $\mathrm{EuH}_{2}$ film, (c) set of optical absorbance spectra $A_{\mathrm{H} / \mathrm{Eu}}(\lambda)$ determined in situ for selected hydrogen contents.

formation. The evident effect of the hydrogen uptake is the decrease of the film absorbance. As a consequence a new maximum is formed during hydride formation in the visible wavelength range around $450 \mathrm{~nm}$. This peak is responsible for the change of color of the film during hydrogen uptake from blue-metallic, characteristic for pure $\mathrm{Eu}$, to the intensive orange of $\mathrm{EuH}_{2}$. It is important to notice that the origin of this maximum is not obvious, since its trace is also marked in the absorbance spectra obtained for low hydrogen contents and even for pure Eu film. The successive hydrogen uptake and associated decrease of absorbance level make this maximum more pronounced and slightly shift its position to a lower wavelength from 500 to $450 \mathrm{~nm}$. The next interesting feature is a locally existing - only in the ultraviolet range (200$350 \mathrm{~nm}$ ) - increase of the film absorbance with hydrogen content, observed for $\mathrm{H} / \mathrm{Eu}$ equal to 1.1 or higher. As a consequence an isosbestic point is formed in the spectra at $310 \mathrm{~nm}$ for these higher hydrogen concentrations, indicating the existence of two phases influencing the thin film's optical properties (solid solution of hydrogen of metallic character and semiconducting dihydride).

The changes of resistance and relative transmittance in thin Eu film (50 nm thick) caused by successive hydrogen doses introduced into the reactor at $298 \mathrm{~K}$ are presented in Fig. 2, while Fig. 3 shows the courses of corresponding equilibrium values as a function of average hydride stoichiometry in the film. The observed relations for Eu are simpler in comparison to those previously described for hexagonal RE metals ( $\mathrm{Gd}[12], \mathrm{Tb}[13])$. This is a consequence of the simpler phase relation of Eu hydrides
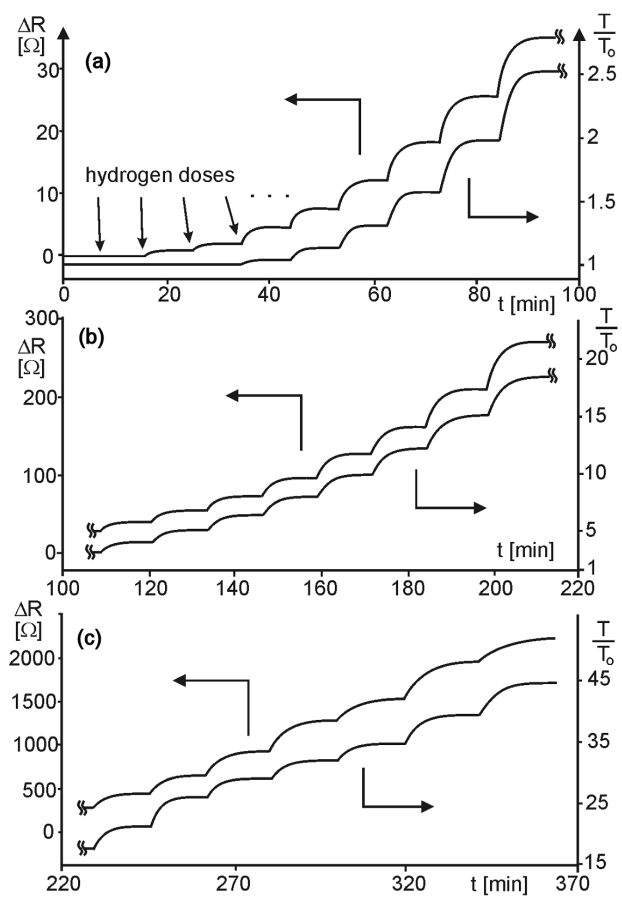

Fig. 2. Course of time-dependent resistance and relative transmittance changes of thin Eu film registered in situ during successive $\mathrm{H}_{2}$ doses introduction into the reactor at $298 \mathrm{~K}$. Division into sectors (a)-(c) better illuminates the progress of the reaction. The interval of $\mathrm{H} / \mathrm{Eu}$ ratio corresponding to every section: (a) $0-0.7$; (b) $0.7-1.4$; (c) $1.4-2.0$.

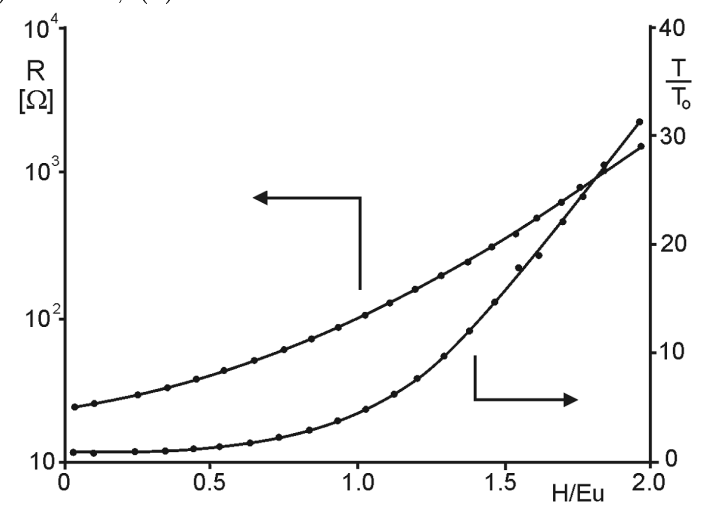

Fig. 3. Dependence of resistance and relative optical transmittance of thin $\mathrm{EuH}_{x}(0<x<2)$ film on hydrogen concentration under equilibrium conditions reached in the reactor after every successive $\mathrm{H}_{2}$ dose introduction at $298 \mathrm{~K}$.

where the metal forms one metallic (solid solution of hydrogen) and one semiconducting $\left(\mathrm{EuH}_{2}\right)$ phase. Therefore, introduction of each $\mathrm{H}_{2}$ dose leads to an increase of both electrical resistance and optical transmittance of the film in the whole range of hydride stoichiometry, as a consequence of the increased content of semiconducting dihydride in the film. Moreover, one can notice that the increase of the equilibrium film transmittance begins from $\mathrm{H} / \mathrm{Eu}$ atomic ratios even below 0.5 , which is much lower than required for the stoichiometric dihydride. 
Both these observations suggest that hydride formation is not a homogeneous process in the film and even for such low average hydrogen content, precipitates of the transparent dihydride are formed locally around some active places in the thin film, changing its global optical properties.

Finally, it is interesting to summarize that in the case of Eu we do not observe a decrease of resistance with hydrogen content which is a characteristic behavior of hydrides of hexagonal $\mathrm{RE}$ metals in the middle range of their stoichiometry. This indicates that this phenomenon is a direct consequence of the existence of a metallic dihydride ( $\beta$ phase) for these metals and its influence on the electrical resistance of the film. This metallic dihydride phase is typical for RE hexagonal metals and is not formed for europium.

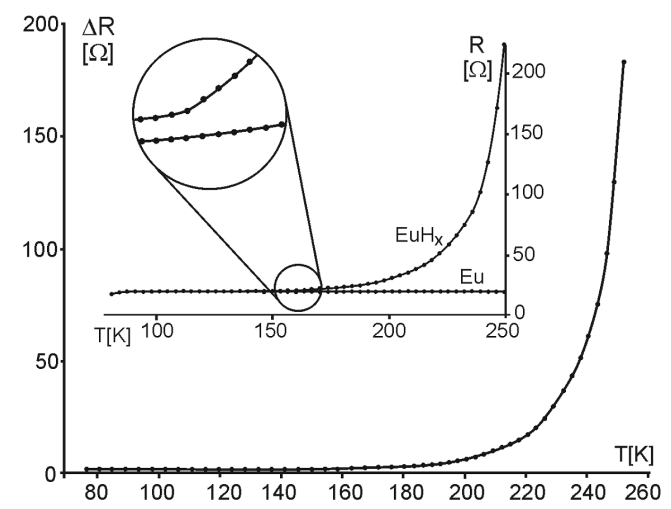

Fig. 4. Dependence of temperature on the resistance of thin Eu film interacting with $\mathrm{H}_{2}$. The main curve shows a difference between curve $\mathrm{EuH}_{x}$ and curve Eu corresponding to net changes of the film resistance caused by $\mathrm{H}_{2}$. Curve $\mathrm{EuH}_{x}$ represents the resistance changes of the $\mathrm{Eu}$ film (maintained primarily in vacuum at $78 \mathrm{~K}$ in the reactor) resulting from the interaction with large dose of hydrogen during the temperature increase. Curve Eu shows the temperature dependence of Eu film under UHV conditions.

The interaction of thin Eu film with hydrogen is different at low temperature. A separate experiment at $78 \mathrm{~K}$ indicates a lack of hydrogen uptake and no changes in the film's resistance. This is an analog of molecular hydrogen interaction with gold at $78 \mathrm{~K}$ [23]. In both systems the lack of adsorption is expected at low temperature and explained by low energy of molecular hydrogen (below activation energy) preventing its dissociation on the surface of these metals. Therefore, an interesting question arises about the temperature limit from which hydrogen is able to incorporate into the bulk of the film and form a hydride. To find an answer, the resistance of $\mathrm{Eu}$ film in the reactor filled with hydrogen $(10 \mathrm{~Pa})$ and disconnected from the pumps was monitored during progressively increased temperature from 78 to $260 \mathrm{~K}$. The obtained course was then corrected by the appropriate dependence obtained in a "blank" experiment with the same film kept in a vacuum. Hence Fig. 4 shows the net effect of $\mathrm{H}_{2}$ interaction with Eu film during heating (the independent courses obtained with (marked by $\mathrm{EuH}_{x}$ ) and without $(\mathrm{Eu})$ hydrogen in the reactor are presented in the inset). The result clearly indicates that the difference between values of resistance measured in both experiments begins at $160 \mathrm{~K}$ and progressively increases with temperature. This means that at this temperature hydrogen from the gas phase starts to incorporate into the Eu film and forms a hydride phase in the bulk of the film. For a more detailed discussion it is interesting to see if $160 \mathrm{~K}$ is exactly the minimal temperature enabling hydrogen dissociation on the Eu surface and its adsorption, or if adsorption occurs at lower temperature but the determined value corresponds to disintegration of a low-temperature ordered hydride phase $\left(\alpha^{\prime}\right)$ created only at the subsurface layer. This hydride phase exists for many hexagonal RE metals and strongly inhibits hydrogen penetration into the bulk [5]. The question is, does this phase also exist for europium hydride?

\subsection{Work function changes}

Changes of work function caused by the interaction of successive hydrogen doses with thin $\mathrm{Eu}$ film at $298 \mathrm{~K}$ are presented in Fig. 5. The introduction of the first few doses (four doses marked by (1)-(4), each consisting of $\approx 10^{18}$ hydrogen particles) leads to a decrease of work function. This change indicates that the hydrogen atom is deformed on the Eu surface, as its electron is attracted by the metal, and forms a positively polarized dipole. The growing number of introduced doses resulted in a progressive decrease of work function as a consequence of the accumulation of hydrogen adspecies on the metal surface. Let us note that the period between introductions of the first four doses was not long enough to enable full response of the metal surface and an equilibrium state to be reached.

Equilibrium returned after the introduction of the fifth dose followed by a longer response time. As a result the change of work function disappeared, i.e. the work function returned to the same level as measured for pure metal before hydrogen adsorption. This indicates that the hydrogen adsorbate accumulated during the introduction of five doses completely disappeared from the metal surface.

On the basis of these observations we can therefore expect that each of the first hydrogen doses led to the creation of a negative $\Delta \Phi$ peak which consists of a rapid decrease of work function, as a result of adsorption and formation of positively polarized hydrogen adspecies, followed by its slower increase due to the adsorbate's incorporation from the surface into the bulk. This expectation is exactly fulfilled when the next several doses are observed $((6)-(9))$. However, the increase of the $\mathrm{H} / \mathrm{Eu}$ atomic ratio in the film changes the film's response to the opposite one. Therefore, tracking of work function changes caused by the introduction of subsequent doses $((10)-(18))$ shows a continuous transition of negative $\Delta \Phi$ peaks into positive ones reflecting the inversion of polar- 


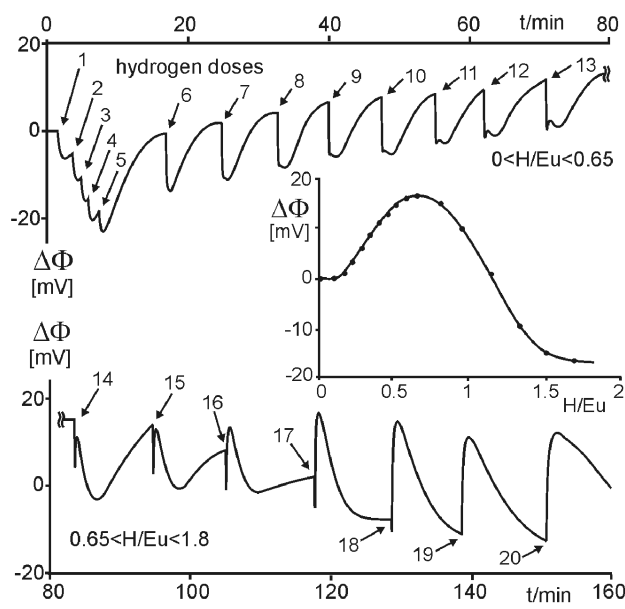

Fig. 5. Course of work function changes in the process of $\mathrm{EuH}_{x}$ formation at $298 \mathrm{~K}$. Hydrogen was introduced in calibrated doses into the static capacitor with thin Eu film. The inversion of polarization of the hydrogen adsorbate is clearly visible. Curve in the inset illustrates the resulted dependence of work function changes at equilibrium on the $\mathrm{H} / \mathrm{Eu}$ average atomic ratio.

ization of the hydrogen adspecies from positively to negatively polarized. As is evident from the presented course, the surface response in a wide range of hydrogen content is a superposition of two opposite $\Delta \Phi$ peaks, indicating the coexistence of two forms of hydrogen adspecies on the surface. Finally, the positive $\Delta \Phi$ peak is a dominant response for higher $\mathrm{H} / \mathrm{Eu}$ concentration (see response after introduction of dose (19)). Its creation proves that the negatively polarized hydrogen adspecies is also formed on the surface and, similarly as the positively polarized adspecies, incorporates into the bulk of the film.

This inversion of charge-transfer direction leading to the formation of two hydrogen adspecies on the surface is a consequence of the evolution of the surface properties, reflected by work function changes at equilibrium state, simultaneously occurring during hydride formation. In this case we focus on the work function changes determined for each hydrogen dose after incorporation of the adsorbate and stabilization of a new equilibrium level. In the discussed course (Fig. 5) this corresponds to the shift of the $\Delta \Phi$ baseline caused by the introduction of each hydrogen dose and the resulting increase of $\mathrm{H} / \mathrm{Eu}$ concentration. It is evident from the plot that the direction of this shift is changed to the opposite with the hydrogen content. The baseline of $\Delta \Phi$ firstly increases with the number of $\mathrm{H}_{2}$ doses for lower $\mathrm{H} / \mathrm{Eu}$ concentration, corresponding to the formation and incorporation of positively polarized hydrogen adspecies (doses up to (13)), then for higher $\mathrm{H} / \mathrm{Eu}$ it decreases when negatively polarized adspecies begin to be dominant on the surface (doses from (14) to (20)). The observed behavior reflects changes of "mirror potential" which is formed directly on the film surface due to the incorporation of appropriate hydrogen adspecies into the bulk. Hence, the resultant accumulation of the positively polarized adspecies in the subsurface region induces negative excess charge (negative potential) on the surface. This is the reason for the observed increase of equilibrium $\Delta \Phi_{\mathrm{eq}}(\mathrm{H} / \mathrm{Eu})$ in the range of lower hydrogen content. The evolution of material properties during hydride formation also changes the conditions of the surface for adsorption. For higher hydrogen content this results in the inversion of polarization of the hydrogen adspecies. Then incorporation of the negatively polarized adsorbate in the subsurface induces positive "mirror potential" decreasing the work function.

The inset of Fig. 5 illustrates the resultant dependence of the equilibrium work function on hydrogen content in the film. The course is characterized by a maximum for the $\mathrm{H} / \mathrm{Eu}$ atomic ratio around 0.7 . It is interesting to note that the change of work function is symmetrical and varies with hydrogen content from +18 to $-18 \mathrm{mV}$ with respect to pure metal film, reflecting the symmetrical surface effect caused by the accumulation of positively and negatively polarized hydrogen adspecies, respectively, in the subsurface region.

The continuous observation of work function changes during inversion of polarization of hydrogen adspecies enables us to analyze and compare the shape of $\Delta \Phi(t)$ peaks formed by two forms of the adsorbate. In the middle range of the $\mathrm{H} / \mathrm{Eu}$ atomic ratio both forms coexist on the surface and the registered signal is a sum of overlapping positive and negative peaks. Let us turn our attention to the complex shape of the front of the response (Fig. 5, doses (14)-(16)). This shape indicates that the negative peak is characterized by faster formation than the positive one. As a consequence, the extremes of the peaks (minimum of the negative peak and maximum of the positive one) are mutually shifted, reflecting differences in kinetics of corresponding surface processes. This suggests that the rate of formation of positively polarized hydrogen adspecies is higher than that of the negatively polarized ones.

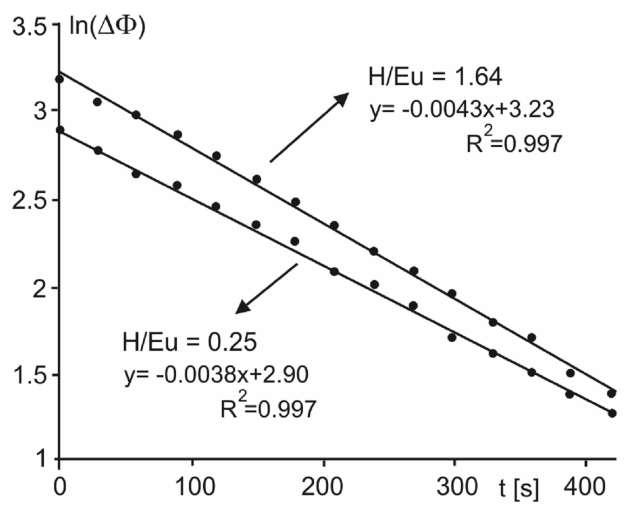

Fig. 6. Examination of work function changes caused by the hydrogen adsorbate incorporation into the bulk of $\mathrm{EuH}_{x}$ film at $298 \mathrm{~K}$ (for average hydrogen content $\mathrm{H} / \mathrm{Eu}$ equal to 0.25 and 1.64 ), according to the first-order kinetic equation. 
By analogy, the decay of the $\Delta \Phi$ signal in the peaks is directly related to the loss of adsorbate from the surface due to its incorporation into the bulk. The quantitative analysis confirms that this process occurring for both the positively (dose (7)) and negatively (dose (19)) polarized hydrogen adsorbate is well described by the first-order kinetic equations, as presented in Fig. 6. The slightly higher slope of linear dependence of $\ln (\Delta \Phi)$ on time for negatively polarized hydrogen adspecies (dominated for higher hydride stoichiometry) as compared with the positively polarized ones indicates that the negative form incorporates faster from the surface into the bulk of the film. This difference in the rate of incorporation can be linked with expected weaker bonding of hydrogen in semiconducting dihydride than in metallic medium of solid solution in the host-metal lattice.

\section{Conclusions}

1. Thin europium film interacting in situ with $\mathrm{H}_{2}$ easily forms hydrides $\mathrm{EuH}_{x}(0<x<2)$ at room temperature and hydrogen pressure around $30 \mathrm{~Pa}$. However, at low temperature $78 \mathrm{~K}$ under these conditions the uptake of hydrogen is not measurable.

2. In the course of $\mathrm{EuH}_{2}$ formation, the light-reflecting metallic thin $\mathrm{Eu}$ film is transformed into a transparent semiconductor. Both the electrical resistance and optical transparency of the film increase with hydride stoichiometry as a consequence of the increased content of semiconducting dihydride in the film. These changes were correlated with the evolution of absorbance spectra in the wavelength range of $200-1100 \mathrm{~nm}$, measured in situ.

3. The lack of hydrogen uptake during $\mathrm{H}_{2}$ interaction with Eu film at $78 \mathrm{~K}$ can be explained by the insufficient energy of molecular hydrogen to dissociate and adsorb on the Eu surface. The measured temperature dependence of the resistance of $\mathrm{Eu}$ film interacting with $\mathrm{H}_{2}$ indicates that hydrogen incorporates from the gas phase into the bulk and forms hydrides at temperatures above $160 \mathrm{~K}$.

4. The transition of hydrogen adsorbate on the Eu surface from positively to negatively polarized form was observed continuously during hydrogen uptake at room temperature. This observation indicates a change in the charge-transfer direction in the hydrogen-europium system.

5. The surface processes associated with $\mathrm{EuH}_{x}$ formation are well described by the first-order kinetic equations. The negatively polarized hydrogen adspecies is characterized by slower formation on the Eu surface but faster incorporation from the surface into the bulk of the film as compared with the positively polarized ones.

\section{Acknowledgments}

This work was supported by the Polish Ministry of Science and Higher Education through grant N N204 241634 (2008-2011).

\section{References}

[1] J.H. Huiberts, R. Griessen, J.H. Rector, R.J. Wijngaarden, J.P. Dekker, D.G. de Groot, N.J. Koeman, Nature 380, 231 (1996).

[2] P. van der Sluis, M. Ouwerkerk, P.A. Duine, Appl. Phys. Lett. 70, 3356 (1997).

[3] J.H. Huiberts, J.H. Rector, R.J. Wijngaarden, S. Jetten, D. de Groot, B. Dam, N.J. Koeman, R. Griessen, B. Hjörvarsson, S. Olafsson, Y.S. Cho, J. Alloys Comp. 239, 158 (1996).

[4] G.G. Libowitz, A.J. Maeland, Handbook on the Physics and Chemistry of Rare Earths, Vol. 3 North-Holland, Amsterdam, 1979, Ch. 26.

[5] P. Vajda, Handbook on the Physics and Chemistry of Rare Earths, Vol. 20, North-Holland, Amsterdam 1995, Ch. 137.

[6] R.R Arons in: Rare Earth Hydrides; Landolt-Bornstein New Series, Vol. 12c, Ch. 63, Ed. K.H. Hellwedge, Springer-Verlag, Berlin 1982, DOI $10.1007 / \mathrm{b} 19987$.

[7] R.R. Arons, in: Rare Earth Hydrides; Landolt-Bornstein New Series, Vol. 19d1, Ch. 23, Ed. H.P.J. Wijn, Springer-Verlag, Berlin 1991, DOI $10.1007 / \mathrm{bb} 38356$.

[8] F.J.A. den Broeder, S.J. van der Molen, M. Kremers, J.N. Huiberts, D.G. Nagengast, A.T.M. van Gogh, W.H. Huisman, N.J. Koeman, B. Dam, J.H. Rector, S. Plota, M. Haaksma, R.M.N. Hanzen, R.M. Jungblut, P.A. Duine, R. Griessen, Nature 394, 656 (1998).

[9] W.E. Vargas, D.E. Azofeifa, N. Clark, J. Phys. D, Appl. Phys. 42, 015416 (2009).

[10] D.E. Azofeifa, N. Clark, W.E. Vargas, Phys. Status Solidi B 242, 2005 (2005).

[11] D.E. Azofeifa, W.E. Vargas, N. Clark, H. Solis, J. Alloys Comp., 446-447, 522 (2007).

[12] E. Nowicka, R. Nowakowski, R. Duś, Appl. Surf. Sci. 254, 4346 (2008).

[13] M. Knor, R. Nowakowski, E. Nowicka, R. Duś, Langmuir 26, 3302 (2010).

[14] M. Knor, R. Nowakowski, R. Duś, Appl. Surf. Sci. 257, 8241 (2011).

[15] J.M. Haschke, M.R. Clark, High Temp. Sci. 7, 152 (1975).

[16] H. Kohlmann, K. Yvon, J. Alloys Comp. 299, L16 (2000).

[17] T. Matsuoka, H. Fujihisa, N. Hirao, Y. Ohishi, T. Mitsui, R. Masuda, M. Seto, Y. Yoda, K. Shimizu, A. Machida, K. Aoki, Phys. Rev. Lett. 107, 025501 (2011).

[18] S. Weber, J. Schoenes, J. Appl. Phys. 102, 113110 (2007).

[19] E. Nowicka, R. Duś, Surf. Sci. 144, 665 (1984).

[20] A. Bachtin, Vacuum 35, 519 (1985).

[21] T. Delchar, A. Eberhagen, F.C. Tompkins, J. Sci. Instrum. 40, 105 (1963).

[22] R. Duś, E. Nowicka, Langmuir 16, 10258 (2000).

[23] R. Duś, E. Nowicka, R. Nowakowski, Langmuir 20, 9138 (2004). 